\title{
Online Adaptive NeuroFuzzy based SVC control strategy for damping low frequency oscillations in multi-machine power system
}

\author{
Saima Ali, Shahid Qamar, Laiq Khan, Umer Akram \\ Department of Electrical Engineering, COMSATS Institute of Information Technology, Abbottabad, Pakistan \\ Email address: \\ saimaali@ciit.net.pk(S. Ali), shahidqamar@ciit.net.pk(S. Qamar), umer00.akram@gmail.com(U. Akram)
}

\section{To cite this article:}

Saima Ali, Shahid Qamar, Umer Akram. Online Adaptive Neurofuzzy Based SVC Control Strategy for Damping Low Frequency Oscillations in Multi-Machine Power System. International Journal of Energy and Power Engineering. Vol. 2, No. 4, 2013 , pp. $172-183$. doi: $10.11648 /$ j.ijepe.20130204.15

\begin{abstract}
An online auxiliary control was designed for Static Var Compensator (SVC) to improve the poorly damped oscillations in multi-machine power system subjected to small and large disturbances. This paper presents auxiliary control based on Adaptive NeuroFuzzy (ANF) control using triangular membership function. Such a model free based control does not require any prior information about the system and is robust to system changes quickly. To minimize the cost function and to tune the parameters of the antecedent and consequent part of the proposed control, a Gradient Descent (GD) learning algorithm is used. The time domain simulation results were carried out for two machine test system for four different cases. In order to exploit the performance and robustness of ANF control, the results were compared with conventional PI and no control. Simulation results and performance indices reveal that the proposed control outperforms during various fault conditions and hence improves the transient stability to a great extend.
\end{abstract}

Keywords: Static Var Compensator, Muti-Machine Power System, Adaptive Neurofuzzy, Triangular Membership Function, Gradient Descent Learning Algorithm

\section{Introduction}

For years, it has been observed that transient stability and damping of low frequency electromechanical oscillations of complex power system can be improved by providing appropriate shunt compensation. Shunt compensation changes the electrical characteristics of the network by injecting reactive power and thus make it more compatible with the changing system conditions [1]. Reasons for low frequency oscillations are sudden load changes, line switching and bulk power transmission over long distances etc. As a result some synchronous generators in interconnected system force to accelerate and some to decelerate against each other in the same vicinity or distant location, creating a speed mismatch among them. Electromechanical oscillations are either inter-area or local mode, ranging from 0.1 to $0.7 \mathrm{~Hz}$ and 0.7 to $2 \mathrm{~Hz}$, respectively [2]. If efficient damping control mechanism is not provided, these oscillations start to grow up with time and reduce the power transfer capacity of lines by demanding higher safety margins [3]. Conventionally,
Power System Stabilizers (PSS) were used to damp out the electromechanical oscillations but these stabilizers do not give satisfactory damping in inter-area mode [4-5]. When system operating condition changes vigorously, PSS was not able to cope with these changes as it is designed for a particular operating point.

The developments in the field of power electronics technologies have resulted in the use of Flexible AC Transmission System (FACTS) controllers in power system. With the introduction of FACTS controllers, transmission lines can be loaded up to its thermal limits and therefore avoid installation of new transmission lines. FACTS controllers with an appropriate external control design have a great potential to efficiently improve the poorly damped oscillations [6-8]. The main FACTS controllers are: Static Var Compensator (SVC), Static synchronous Compensator (STATCOM), Thyristor Controlled Series Capacitor (TCSC), Phase Shifting Transformer (PST), Static Synchronous Series Comparator (SSSC) and Thyristor Controlled Series Reactor (TCSR).

SVC is one of the most commonly used FACTS controller which helps in providing fast reactive shunt compensation 
on transmission lines [9]. SVC controls reactive power by controlling the susceptance of passive devices. System voltage is controlled by controlling the reactive power and hence indirectly active power is controlled which results in damping of electromechanical oscillations [10-15]. Damping of electromechanical oscillations can be achieved through designing of appropriate external control for SVC. Proportional Integral (PI) and Proportional Integral Derivative (PID) controllers are the most frequently used conventional techniques available as an SVC external control. PI controller is the other commonly used scheme $[16,17]$. Although the PI controllers present ease and simplicity of design, but their operating condition becomes less effective when the system conditions vary extensively or large disturbances take place [18]. To circumvent these drawbacks, recently, Fuzzy Logic Controllers (FLCs) and Artificial Neural Network Controllers (ANNCs) have been used for oscillations damping control in the power systems [19-23]. But majority of these artificial intelligence based control for SVC are designed for linearized power system and its control parameters are updated off-line. As power system is highly nonlinear and any vagueness in the system can drastically change its operating point. So these linear control designs may not give better performance under such circumstances.

In the majority fuzzy control strategies, constant control gain factor is used for a particular operations condition [24]. One disadvantage of the constant gain factor approaches is that the controller designed under a certain situation might become less efficient for other operating conditions due to unnecessary and excessive control action. In [25, 26], a fuzzy logic and an adaptive fuzzy logic based damping control approach are used for SVC. The advantage of adaptive fuzzy control scheme is that the parameters of fuzzy logic are tuned online according to the changes in the operating condition of the system. Also, a more effective an adaptive neuro-fuzzy control strategies have been used to improve the damping oscillations of the power system by $[27,28]$.

The aim of this paper is to investigate the design of Adaptive NeuroFuzzy based SVC (SVC-ANF) control because it is more robust to change operating characteristics of the system and therefore improve the system damping. ANF control combines the advantages of both fuzzy logic and neural network. The learning capability of neural network is used to tune the parameters of fuzzy logic in different operating conditions to achieve better performance. This approach has learning ability for establishing and updating the fuzzy rules and its parameters continuously. The advantage of this proposed strategy is that the parameters of proposed ANF are updated until the solution converges. In ANF control parameters are updated online without any preliminary knowledge about the network. The parameters involved in SVC need to change with time. So, there is a continuous learning mechanism so that they can adapt their parameters with different conditions. This hybrid ANF strategy increases the robustness, efficiency, convergence and efficiency of the system. The productivity and computational ability of the proposed ANF technique is better than the aforementioned control strategies. Results are tested by installing SVC having ANF as a supplementary controller on the two machine three bus bar system. To validate the performance of proposed SVC-ANF control, the time domain simulations are compared with those of conventional SVC-PI and SVC itself.

This paper is organized as follows; section 2 presents SVC transient stability model, steady-state characteristics and its internal control. In section 3, mathematical modeling of SVC installed with multi-machine power system is presented. Section 4 discusses the ANF control design; layered architecture and parameter update rules. Finally, simulation results and conclusion are discussed in section 5 and 6 .

\section{SVC Transient Stability Model and Control}

An SVC is shunt connected FACTS controller which generates and absorbs reactive power in order to control specific parameters of power system. The compensator shown in Fig. 1 is of TSC-TCR type and it usually consists of nTSC banks and a single TCR connected in parallel. TSC banks are either switched in or out, generating leading Vars in stepwise fashion. While in TCR, the current through the reactor is phase controlled and can be varied from zero to maximum to continuously controlled the lagging Vars. By combining the operations of both TSC and TCR a continuous control of reactive power is achieved. When system voltage is less SVC behaves like a capacitor and generates leading vars. While when system voltage is high SVC behaves like a reactor and absorbs reactive power (generates lagging Vars).

\subsection{SVC Phasor Model}

The transient stability model of SVC under balanced three phase AC system voltages can be represented as [29];

$$
\begin{gathered}
\bar{I}_{a b c_{-} s c v}=-B_{s v c} \bar{e}_{a b c_{-} s v c} \\
\bar{I}_{a b c_{-} s c v}=\bar{I}_{T C R}\left(\psi_{s v c}\right)+\bar{I}_{T S C}
\end{gathered}
$$

Where, $\bar{I}_{s v c}$ is the current injected by SVC and $\bar{e}_{s v c}$ is the terminal voltage of SVC control.

$$
\bar{I}_{T C R}\left(\psi_{s v c}\right)=-\frac{V}{\omega L}\left(2-\frac{2 \psi_{s v c}}{\pi}+\frac{1}{\pi} \sin 2 \psi_{s v c}\right)
$$

The SVC susceptance is given as

$$
B_{s v c}=B_{T C R}\left(\psi_{s v c}\right)+B_{T S C}
$$

By considering the coupling transformer then 


$$
\begin{gathered}
B_{s v c}=\frac{B_{\sigma}\left(B_{T C R}\left(\psi_{s v c}\right)+B_{T S C}\right)}{B_{T C R}\left(\psi_{s v c}\right)+B_{T S C}+B_{\sigma}} \\
B_{T C R}\left(\psi_{s v c}\right)=\frac{1}{\omega L}\left(2-\frac{2 \psi_{s v c}}{\pi}+\frac{1}{\pi} \sin 2 \psi_{s v c}\right) \\
B_{T S C}=n B_{C}
\end{gathered}
$$

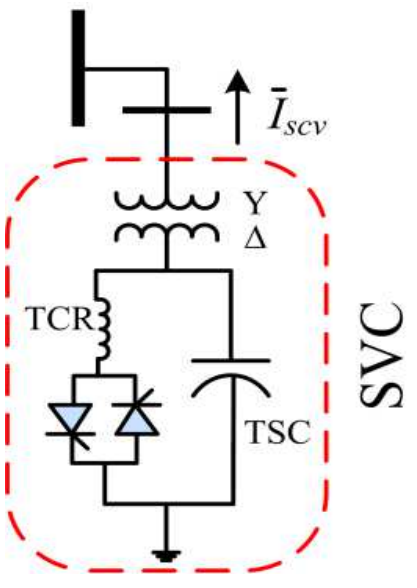

Figure1. TSC-TCR Type SVC

where $\psi_{s v c}=\phi+\varphi$, with ' $\phi$ ' as a synchronizing angle and ' $\varphi$ ' is the firing angle and lies in the range of $90^{\circ}$ to $180^{\circ}$. When $\psi_{s v c}=90^{\circ}$, SVC susceptance is maximum while when $\psi_{s v c}=180^{\circ}$, SVC susceptance is zero. The reactive power injected by SVC is given by

$$
Q_{i n j_{-} s v c}=-e_{m}{ }^{2} B_{s v c}
$$

By using $d q$ reference frame, the current injected by SVC control can be written as follows;

$$
\bar{I}_{d q_{-} s v c}=\frac{2}{3}\left[\begin{array}{lll}
1 & a & a^{2}
\end{array}\right]\left[\begin{array}{l}
I_{a_{-} s v c} \\
I_{b_{-} s v c} \\
I_{c_{-} s v c}
\end{array}\right], \text { where } a=e^{j \frac{2 \pi}{3}}
$$

\subsection{SVC Characteristics}

SVC V-I steady state characteristics [30] are shown in Fig. 2.

The conditions for operation of SVC in different modes are as follow:

- In voltage regulation mode, SVC susceptance is

$$
-B_{c \max }<B_{s v c}<B_{l \max } \text { and } e_{s v c}=e_{r e f}+I_{s v c} X_{s}
$$

- In fully inductive mode, SVC susceptance is

$$
B_{s v c}=B_{l \max } \text { and } e_{s v c}=\frac{I_{s v c}}{B_{l \max }}
$$

- In fully capacitive mode, SVC susceptance is

$$
B_{s v c}=B_{c \max } \text { and } e_{s v c}=\frac{I_{s v c}}{B_{c \max }}
$$

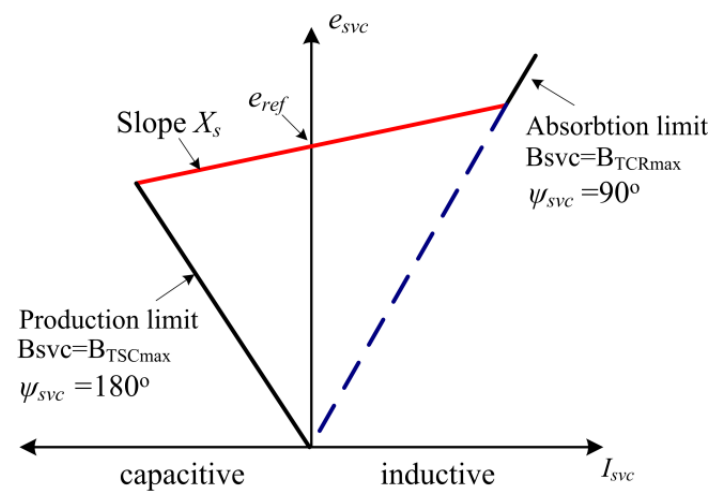

Figure2. SVC V-I Steady-State Characteristics

\subsection{SVC Internal Control}

The Fig. 3 depicts the SVC connected to a three phase positive sequence balanced multi-machine power system along with its internal control. The internal control comprises of measurement unit, supplementary damping control, voltage regulator, synchronizing system and pulse generation module. The purpose of the measurement module is to provide the necessary inputs to other control blocks to perform their functions. Voltage and current is measured using PT's and CT's. Power is measured by multiplying the voltage and current signals. Supplementary damping control used is ANF and is discussed in section 4. Output of measurement block is given as input to the voltage regulator. In voltage regulator it is compared with reference signal and error signal is proceed as input to PI controller. The output of the controller is per unit susceptance which is generated to reduce the error signal to zero. Output of voltage regulator corresponds to the required reactive power output of compensator. Firing angle $(\varphi)$ can be calculated by using susceptance to firing angle converter. The purpose of the synchronizing system is to generate pulses $(\phi)$ that must be synchronized with the fundamental component of system voltage to minimize the harmonics. A commonly used synchronizing system is phase locked loop (PLL).

\section{SVC Installed with Multi-Machine Power System}

The $6^{\text {th }}$ order dynamic model of multi-machine power system used in this article is given as follows;

$$
\begin{aligned}
& \frac{d \delta}{d t}=\omega_{r e f} \omega \\
& M \frac{d \omega}{d t}=P_{m}-P_{e}-D \omega
\end{aligned}
$$




$$
\begin{gathered}
T_{q o}^{\prime} \frac{d E_{d}^{\prime}}{d t}=\left(x_{q}-x_{q}^{\prime}\right) I_{q}-E_{d}^{\prime} \\
T_{d o}^{\prime} \frac{d E_{q}^{\prime}}{d t}=E_{f d}-\left(x_{d}-x_{d}^{\prime}\right) I_{d}-E_{d}^{\prime} \\
T_{q o}^{\prime \prime} \frac{d E_{d}^{\prime \prime}}{d t}=\left(x_{q}-x_{q}^{\prime \prime}\right) I_{q}-E_{d}^{\prime \prime} \\
T_{d o}^{\prime \prime} \frac{d E_{q}^{\prime \prime}}{d t}=E_{q}^{\prime}-E_{q}^{\prime \prime}-\left(x_{d}^{\prime}-x_{d}^{\prime \prime}\right) I_{d}
\end{gathered}
$$

The stator algebraic equations with zero armature resistance are

$$
\left[\begin{array}{l}
\bar{U}_{d} \\
\bar{U}_{q}
\end{array}\right]=\left[\begin{array}{cc}
x_{q}^{\prime \prime} & 0 \\
0 & x_{d}^{\prime \prime}
\end{array}\right]\left[\begin{array}{l}
\bar{I}_{q} \\
\bar{I}_{d}
\end{array}\right]+\left[\begin{array}{c}
E_{d}^{\prime \prime} \\
E_{q}^{\prime \prime}
\end{array}\right]
$$

The terminal voltage of the generators in machine coordinates can be expressed as

$$
\bar{U}_{G}=E_{d}^{\prime \prime}+x_{q}^{\prime \prime} I_{q}+j\left(E_{q}^{\prime \prime}-x_{d}^{\prime \prime} I_{d}\right)
$$

The network model before installation of SVC, between bus bars 1 and 2 is given as;

$$
\left[\begin{array}{c}
0 \\
0 \\
0 \\
\bar{I}_{G}
\end{array}\right]=\left[\begin{array}{llll}
\bar{Y}_{11} & \bar{Y}_{12} & \bar{Y}_{13} & \bar{Y}_{1 G} \\
\bar{Y}_{21} & \bar{Y}_{22} & \bar{Y}_{23} & \bar{Y}_{2 G} \\
\bar{Y}_{31} & \bar{Y}_{32} & \bar{Y}_{33} & \bar{Y}_{3 G} \\
\bar{Y}_{G 1} & \bar{Y}_{G 2} & \bar{Y}_{G 3} & \bar{Y}_{G G}
\end{array}\right]\left[\begin{array}{c}
\bar{U}_{1} \\
\bar{U}_{2} \\
\bar{U}_{K} \\
\bar{U}_{G}
\end{array}\right]
$$

After installation of SVC into the network, the network model can be written as

$$
\left[\begin{array}{ccc}
\bar{Y}_{11}^{\prime} & 0 & \bar{Y}_{1 G} \\
0 & \bar{Y}_{22}^{\prime} & \bar{Y}_{2 G} \\
\bar{Y}_{G 1}^{\prime} & \bar{Y}_{G 2} & \bar{Y}_{G G}
\end{array}\right]\left[\begin{array}{c}
\bar{U}_{1} \\
\bar{U}_{2} \\
\bar{U}_{G}
\end{array}\right]=\left[\begin{array}{c}
-\bar{I}_{1 K} \\
-\bar{I}_{2 K} \\
\bar{I}_{G}
\end{array}\right]
$$

The output voltage of SVC in phasor form is given by;

$$
\bar{e}_{s v c}=e_{m} \angle \psi_{s v c}
$$

From Fig. 4, the voltage difference across the coupling transformer is

$$
\bar{U}_{K}=j x_{c} \bar{I}_{s v c}+\bar{e}_{s v c}
$$

Where,

$$
\bar{I}_{s v c}=\bar{I}_{1 K}+\bar{I}_{2 K}
$$

The voltage at the bus bars 1 and 2 can be written as

$$
\left[\begin{array}{l}
\bar{U}_{1} \\
\bar{U}_{2}
\end{array}\right]=\left[\begin{array}{cc}
j x_{1 K} & 0 \\
0 & j x_{2 K}
\end{array}\right]\left[\begin{array}{l}
\bar{I}_{1 K} \\
\bar{I}_{2 K}
\end{array}\right]+\left[\begin{array}{l}
1 \\
1
\end{array}\right] \bar{U}_{K}
$$

Putting value of $\bar{U}_{K}$ from (26) in (29),

$$
\left[\begin{array}{l}
\bar{U}_{1} \\
\bar{U}_{2}
\end{array}\right]=\left[\begin{array}{cc}
j\left(x_{1 K}+x_{c}\right) & j x_{c} \\
j x_{c} & j\left(x_{2 K}+x_{c}\right)
\end{array}\right]\left[\begin{array}{l}
\bar{I}_{1 K} \\
\bar{I}_{2 K}
\end{array}\right]+\left[\begin{array}{l}
1 \\
1
\end{array}\right] \bar{e}_{s v c}
$$

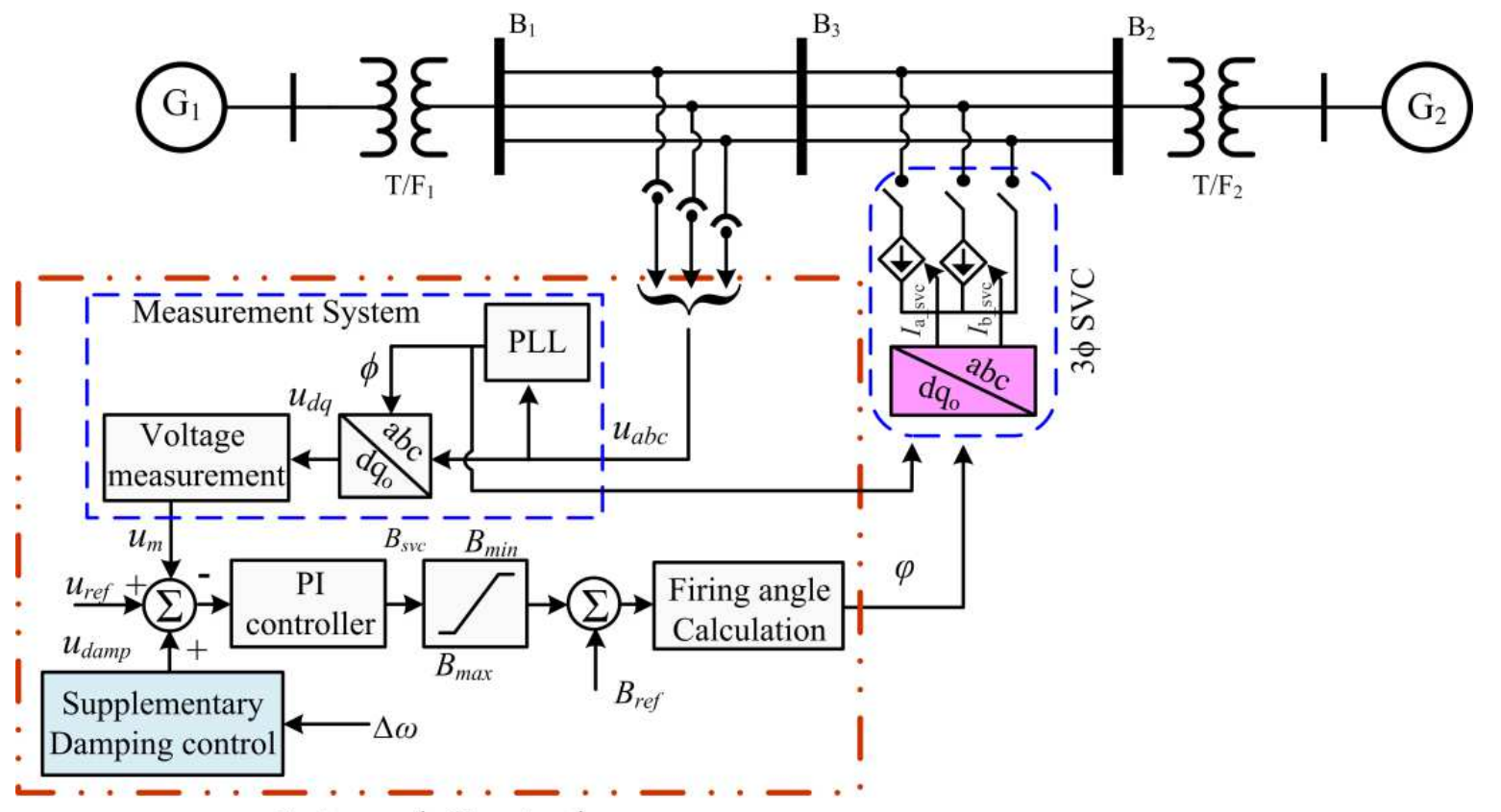

Internal Control

Figure3. Multi-Machine Power System with SVC Internal Control 


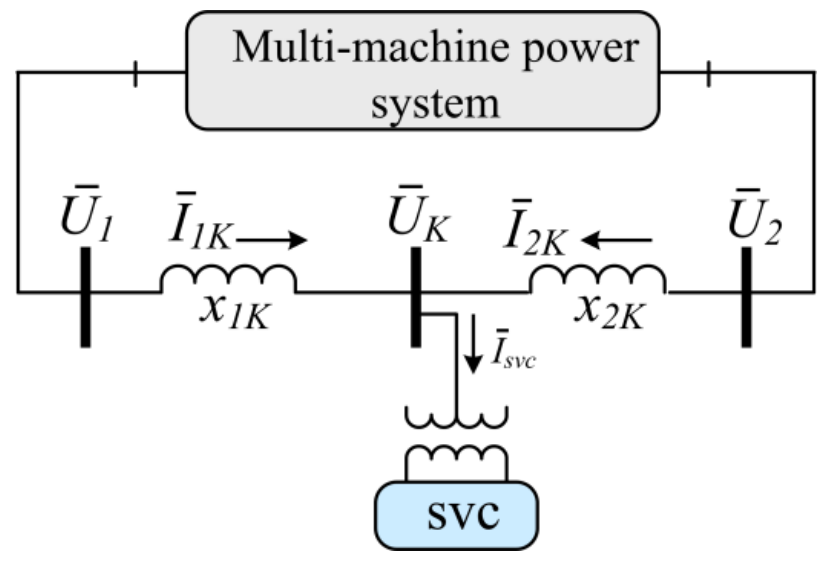

Figure4. SVC Connected Between Bus Bars 1 and 2

$$
\left[\begin{array}{l}
\bar{I}_{1 K} \\
\bar{I}_{2 K}
\end{array}\right]=\frac{1}{X}\left[\begin{array}{cc}
j\left(x_{1 K}+x_{c}\right) & -j x_{c} \\
-j x_{c} & j\left(x_{2 K}+x_{c}\right)
\end{array}\right]\left[\begin{array}{c}
\bar{U}_{1}-\bar{e}_{s v c} \\
\bar{U}_{2}-\bar{e}_{s v c}
\end{array}\right]
$$

Where,

$$
X=x_{c}^{2}-\left(x_{1 K}+x_{c}\right)\left(x_{2 K}+x_{c}\right)
$$

Using (24) the generator current is given as,

$$
\bar{I}_{G}=\left(\bar{Y}_{G G}-\left[\begin{array}{ll}
\bar{Y}_{G 1} & \bar{Y}_{G 2}
\end{array}\right] \bar{Y}_{U_{12}}^{-1}\left[\left[\begin{array}{c}
\bar{Y}_{1 G} \\
\bar{Y}_{2 G}
\end{array}\right]\right) \bar{U}_{G}+\left[\begin{array}{ll}
\bar{Y}_{G 1} & \bar{Y}_{G 2}
\end{array}\right] \bar{Y}_{U_{12}}^{-1}\left[\begin{array}{c}
j \frac{x_{2 K}}{X} \\
j \frac{x_{2 K}}{X}
\end{array}\right] \bar{e}_{s v c}\right.
$$

Where,

$$
\bar{Y}_{U_{12}}^{-1}=\left[\begin{array}{cc}
\bar{Y}_{11}^{\prime}+\frac{j\left(x_{1 K}+x_{c}\right)}{X} & \frac{-j x_{c}}{X} \\
\frac{-j x_{c}}{X} & \bar{Y}_{22}^{\prime}+\frac{j\left(x_{2 K}+x_{c}\right)}{X}
\end{array}\right]
$$

\section{Adaptive NeuroFuzzy Control Design}

The fuzzy system is fundamentally adaptive and nonlinear in nature which provides robust performance for the parameter variations and load disturbances [31, 32]. To efficiently improve the transient stability of the system and to damp the low frequency electromechanical oscillations, online Adaptive NeuroFuzzy (ANF) control is proposed as a SVC auxiliary control. In addition to the prime objective of SVC control to maintain the system voltage at the desired constant level, auxiliary control is used to damp the electromechanical oscillations by providing the reference voltage subject to the changes in the system state. Also, due to these undesired oscillations, the power system becomes uncertain. To overcome these uncertainties and imprecision ANF is very flexible, robust and feasible. Fig. 5 shows the layered architecture of ANF control. It follows the simple fuzzy IF-THEN rules defined in the general form as,

$$
\begin{gathered}
R_{2} \text { : If } x_{12} \text { is } \aleph_{12} \text { and } x_{22} \text { is } \aleph_{22} \text { and ... } x_{j 2} \text { is } \boldsymbol{\aleph}_{j 2} \\
\text { Then } y_{2}=\sum_{j=1}^{k} x_{j} \chi_{j 2}+\beta_{2} \\
R_{1} \text { : If } x_{11} \text { is } \aleph_{11} \text { and } x_{21} \text { is } \aleph_{21} \text { and } \ldots x_{j 1} \text { is } \boldsymbol{\aleph}_{j 1} \\
\text { Then } y_{1}=\sum_{j=1}^{k} x_{j} \chi_{j 1}+\beta_{1} \\
R_{m}: \text { If } x_{1 m} \text { is } \aleph_{1 m} \text { and } x_{2 m} i s \aleph_{2 m} \text { and } \ldots x_{j m} \text { is } \aleph_{j m} \\
\text { Then } y_{m}=\sum_{j=1}^{k} x_{j} \chi_{j m}+\beta_{m}
\end{gathered}
$$

Where, $R_{m}$ is the $m$ th fuzzy rule, $x_{j m}$ is the $j$ th input to $m$ th rule, $\chi_{j m}$ are the weights of the output layer and $\beta_{m}$ is the bias factor of the $m$ th rule. $\boldsymbol{\aleph}_{j m}$ represents the triangular fuzzy membership function used in this article, generally defined as

$$
\boldsymbol{\aleph}_{j m}= \begin{cases}1-2 \frac{a b s\left(x_{j}-\kappa_{j m}\right)}{v_{j m}}, & \kappa_{j m}-\frac{v_{j m}}{2}<x<\kappa_{j m}+\frac{v_{j m}}{2} \\ 0, & \text { otherwise }\end{cases}
$$

Here, $\kappa_{j m}$ and $v_{j m}$ are the mean and standard deviation of the triangular membership function respectively. The firing strength $\vartheta_{m}$ of each $m t h$ rule is computed in (30) using T-norm product operator.

$$
\vartheta_{m}=\prod_{n}\left[\aleph_{1} \aleph_{2} \aleph_{3} \ldots \aleph_{k}\right]
$$

The variable ' $k$ ' defines the number of fuzzy membership functions in a rule. The final centroid defuzzified output of the layered network is given by,

$$
u=\frac{\sum_{p=1}^{n} \vartheta_{p} y_{p}}{\sum_{p=1}^{n} \vartheta_{p}}
$$

Where, $y_{p}=\left[\begin{array}{lllll}y_{1} & y_{2} & y_{3} & \ldots & y_{n}\end{array}\right]$ are the outputs of the neural network defined by (32);

$$
y_{p}=\sum_{p=1}^{n} x_{j} \chi_{j p}+\beta_{p}, \quad p=1,2, \ldots, n
$$

The layered network is primarily divided into input layer, hidden layer and output layer. The network architecture is subdivided into six layer with layer I, layer II and layer III as an antecedent portion and layer IV as a consequent portion. The network layers are identified as; 


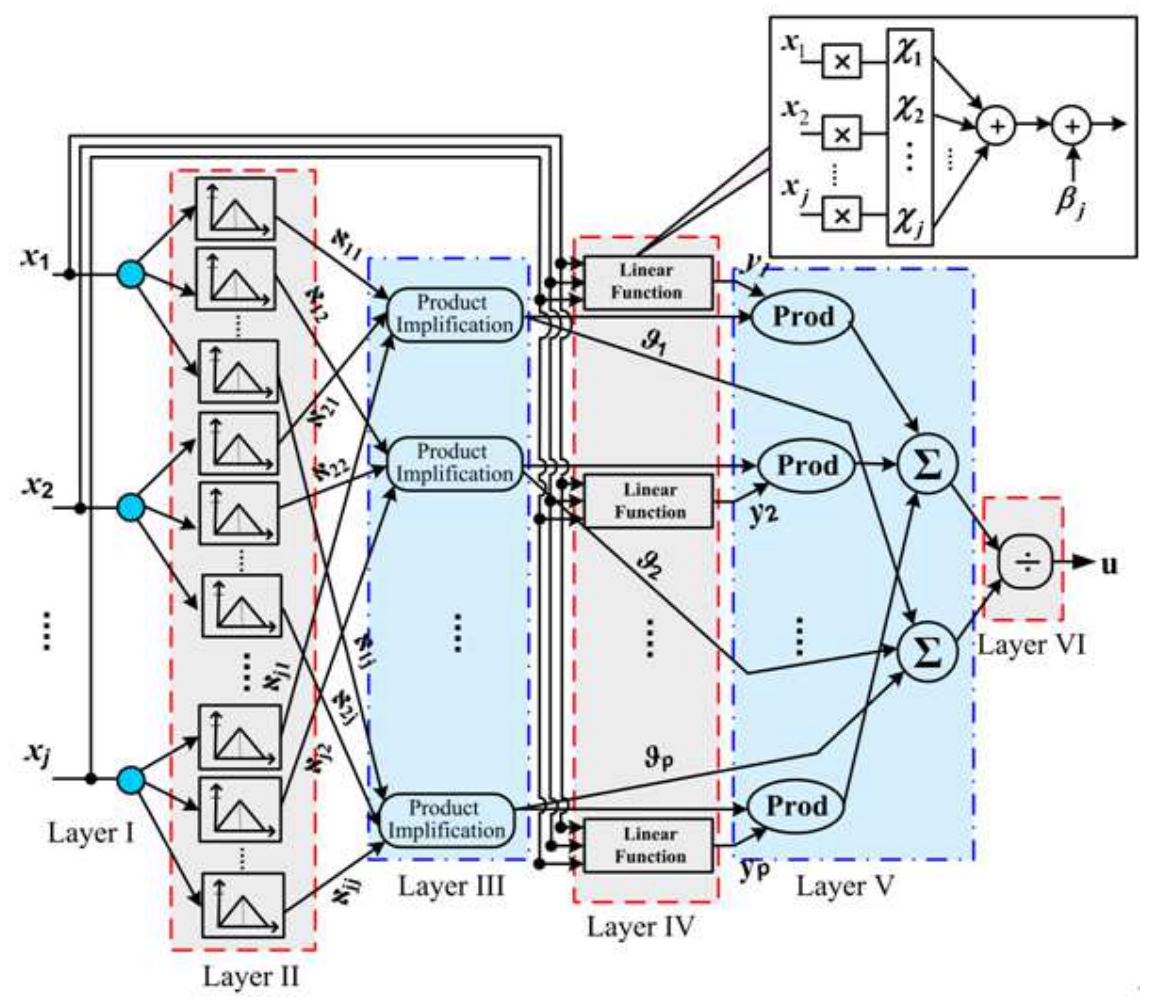

Figure5. ANF Architecture

- Layer I: Input layer.

- Layer II: Inputs are fuzzified into triangular membership function by using (29).

- $\quad$ Layer III: T-norm product operator is calculated by using (30).

- Layer IV: consequent part of ANF outputs is computed by using (32).

- Layer V and layer VI: Computes the defuzzified output by using (31).

The closed loop law of the proposed control along with power system is shown in Fig. 6. In this paper, speed deviation $(\Delta \omega)$ of the generators and its delay are taken as a control input to the ANF control. When disturbances (I) either small or large are added to the system, the parameters of ANF control are updated online and adaptively by using GD algorithm. In response, the ANF control generates the control effort $(u)$ and passes it to the SVC control, where it adds up to the difference of reference and peak value of bus voltage to which it is connected and therefore helps in providing additional damping.

\subsection{Update Parameter Rules}

The parameters estimation is essential for setting up the fuzzy linguistic model of a system to a fixed number of rules. A precise collection of the crisp input data and output data can be expressed to minimize the error between the current output value and the desired value for the neuro-fuzzy system. For this reason, a GD method is used to minimize the cost function. The parameters of the ANF control are updated online using the GD algorithm which is based on the following cost function.

$$
\Lambda=\frac{1}{2} \sum_{i=0}^{M}\left(\omega_{i}-\omega_{r e f}\right)^{2}, M=1
$$

Where, $\omega$ and $\omega_{\text {ref }}$ are the actual and desired value of the plant. The control parameter $\kappa_{j m}, v_{j m}, \chi_{k m}$ and $\beta_{m}$ are updated using the following rules to minimize the cost function $(\Lambda)$.

$$
\begin{gathered}
\kappa_{j m}(t+1)=\kappa_{j m}(t)-\gamma \frac{\partial \Lambda}{\partial \kappa_{j m}} \\
v_{j m}(t+1)=v_{j m}(t)-\gamma \frac{\partial \Lambda}{\partial v_{j m}} \\
\chi_{k m}(t+1)=\chi_{k m}(t)-\gamma \frac{\partial \Lambda}{\partial \chi_{k m}} \\
\beta_{m}(t+1)=\beta_{m}(t)-\gamma \frac{\partial \Lambda}{\partial \beta_{m}}
\end{gathered}
$$

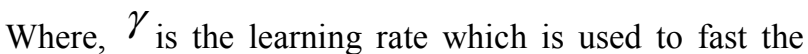
convergence of the system and its value is selected between ' 0 ' and ' 1 ' by using hit and trial method. The partial derivatives w.r.t to control parameters is found using the chain rule as: 


$$
\frac{\partial \Lambda}{\partial \kappa_{j m}}=\left(\omega-\omega_{r e f}\right) \Gamma\left(\frac{2 \operatorname{sign}\left(x_{j}-\kappa_{j m}\right)}{v_{j m}}\right)
$$

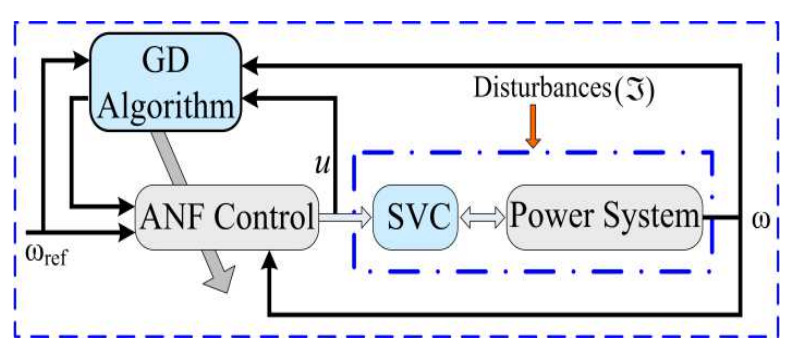

Figure6. Closed Loop of Proposed Control Design

$$
\begin{aligned}
\frac{\partial \Lambda}{\partial v_{j m}} & =\left(\omega-\omega_{r e f}\right) \Gamma\left(\frac{a b s\left(x_{j}-\kappa_{j m}\right)}{v_{j m}{ }^{2}}\right) \\
\frac{\partial \Lambda}{\partial \chi_{k m}} & =x_{j}\left(\omega-\omega_{r e f}\right) \Theta \\
\frac{\partial \Lambda}{\partial \beta_{m}} & =\left(\omega-\omega_{r e f}\right) \Theta
\end{aligned}
$$

Where, $\Gamma=\frac{y_{m}-\omega}{\sum_{p=1}^{n} \vartheta_{m}}$ and $\Theta=\frac{\vartheta_{m}}{\sum_{p=1}^{n} \vartheta_{m}}$

Equation (37) to (40) shows the required change in $\kappa_{j m}, v_{j m}, \chi_{k m}$ and $\beta_{m}$.

\section{Simulation Results and Discussion}

The test system which is used to carry the simulation to access the performance evaluation of the proposed control scheme is shown in Fig. 7 to damp out the electromechanical oscillations. It is based on two-machine, three bus-bar system. The SVC-based ANF control design and time domain simulations are developed in MATLAB SimPower toolbox. The generators ratings are taken as $1400 \mathrm{MVA}\left(\mathrm{G}_{1}\right)$ and 700 MVA $\left(\mathrm{G}_{2}\right)$. The generators are connected to a tie-line through three-phase step up transformers with rating $13.8 / 500 \mathrm{kV}$. The length of transmission line is $500 \mathrm{~km}$ with 200 MVA SVC shunted in the mid of it. To access the simulations load flow is performed by taking machine-1 $\left(\mathrm{M}_{1}\right)$ as a PV generator bus and machine-2 $\left(\mathrm{M}_{2}\right)$ as a swing bus. The initial conditions for $\mathrm{M}_{1}$ and $\mathrm{M}_{2}$ are $970 \mathrm{MW}, 150$ MVars and 363 MW, 432 MVars, respectively. The simulation results of proposed control strategy are compared with that of conventional PI control and without auxiliary control.

To ensure the validation and robustness of the proposed online ANF control scheme, various types of small and large disturbances are added to the test system at different times during simulations. They are characterized into different scenarios as shown in the subsection.

\subsection{Scenario 1}

In the first scenario, a three phase fault is applied on line $\mathrm{L} 1$ at $\mathrm{t}=0.1 \mathrm{sec}$ on the test system as shown in Fig. 7. The duration of the fault is $0.115 \mathrm{sec}$ and it is a self clearing fault. Fig. 8 shows the system response for this severe fault. It is obvious from the results that SVC-ANF control is much more successful in damping the power oscillations as compared to SVC-PI control and without control and brings the system back to its original steady state condition. In case of SVC-ANF control, the performance of speed deviation ( $\omega 21)$, shown in Fig. 8(a) is improved by $47 \%$ and $63 \%$ as compared to SVC-PI control and without control, respectively in terms of settling time. The effectiveness of proposed controller in terms of rotor angle deviation is depicted in Fig. 8(b). It shows that the ANF control quickly stabilizes the rotor angle to its nominal steady state condition. Fig. 8(c) shows the active power flow in line L1. Here, less oscillation has been observed in case of SVC-ANF control after post fault as compared to SVC-PI control and without control. From all the above discussion, it is analyzed that the ANF shows good regulation, has more computational ability, robustness and effectiveness than PI controller.

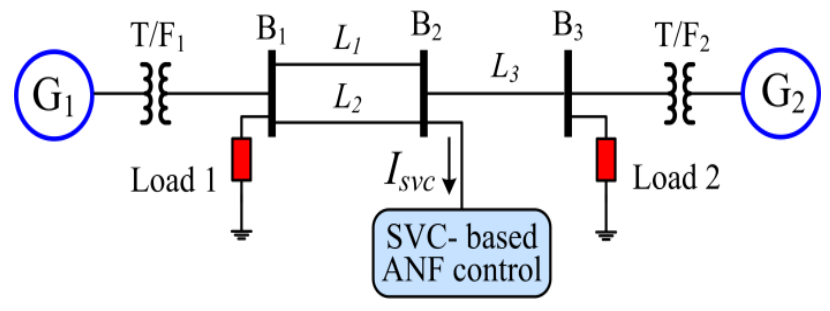

Figure7. Two Machines Test System

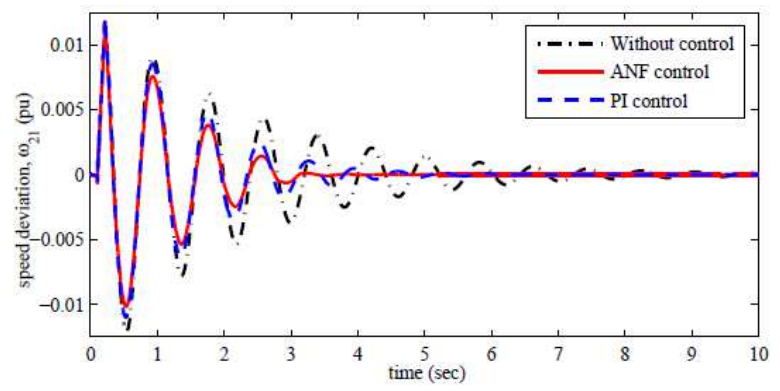

(a) Speed Deviation, $\omega_{21}$

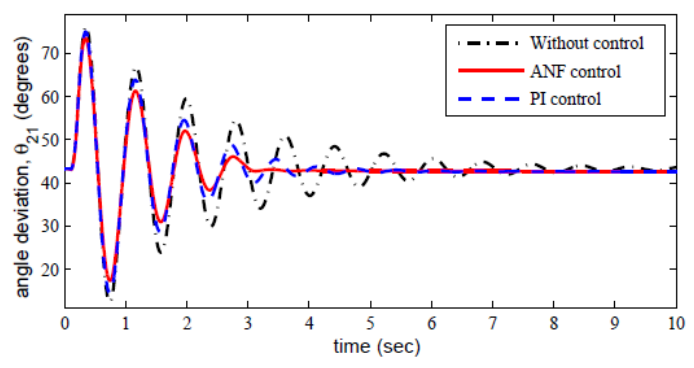

(b) Angle Deviation, $\theta_{21}$ 


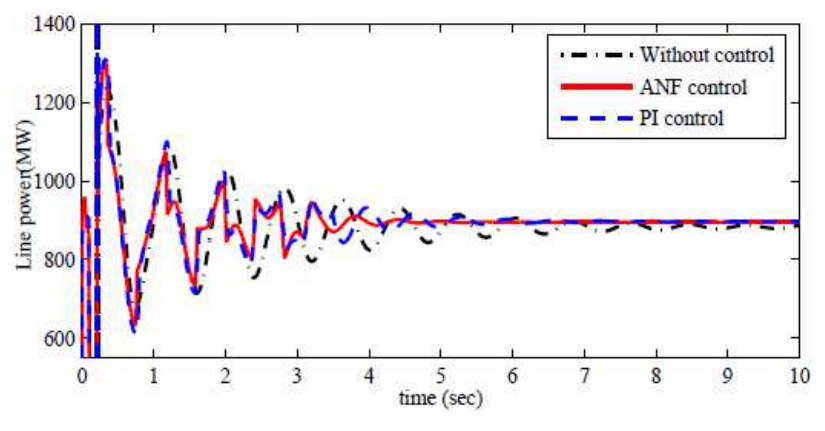

(c) Line Power Injected

Figure8. Scenario 1 Simulation Results

\subsection{Scenario 2}

This scenario constitutes the application of multiple three-phase faults at different locations in the test system in order to check the performance of the proposed control scheme. The first three-phase, self clearing fault is applied on line $L_{2}$ at $t=0.1 \mathrm{sec}$ and another on line $\mathrm{L}_{3}$ at $\mathrm{t}=7 \mathrm{sec}$ for $115 \mathrm{msec}$. The time domain simulation results for this type of faults are shown in Fig. 9 from where it is evident that ANF control has improved the performance of system with less settling time and overshoots as compared to SVC-PI and without auxiliary control. A performance improvement of $20 \%$ and $33 \%$ is achieved in terms of settling time for speed variation as compared to SVC-PI and without control, respectively as shown in Fig. 9(a). The improvement in rotor angle stability can be seen from Fig. 9(b). Here again the rotor angle settles to its original steady state after application to two severe three-phase faults. When no auxiliary control is applied to SVC, the power oscillations are poorly damped after post fault as shown in Fig. 9(c).

\subsection{Scenario 3}

In this scenario, a three-phase fault is applied at line $\mathrm{L}_{2}$ at $\mathrm{t}=0.1 \mathrm{sec}$. The fault is cleared by permanently removing the line $\mathrm{L}_{2}$. Fig. 10 shows that SVC-ANF control contributes more efficiently to damp the system oscillations as compared to SVC-PI and without auxiliary control and brings the system to a new steady-state. It is clear from Fig. 10(a), that the SVC-ANF control attains a performance improvement of $40 \%$ and $60 \%$ in terms of settling time, for speed deviation of the rotor as compared to SVC-PI and without control, respectively. Fig. 10(b) reveals the rotor angle deviation shifted to a new steady state. Furthermore, the active power of the tie-line has a degraded response in the steady-state region in case of SVC-PI and without control as shown in Fig. 10(c). From above discussions, it is observed that the ANF shows good regulation, robustness, efficiency, and gives better computational ability than PI controller. Also the proposed ANF based SVC have increases the stability and productivity of the power system and provide the better convenience to the system in this scenario.

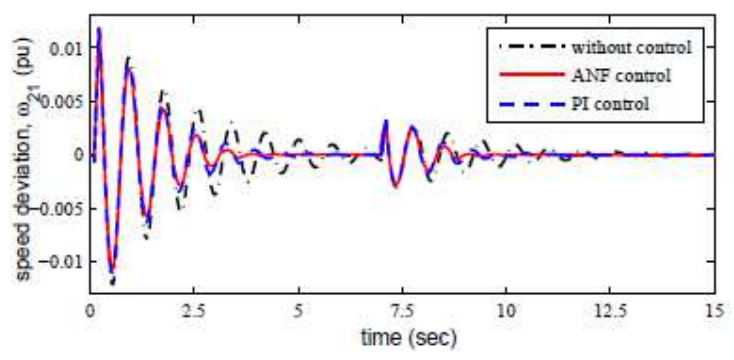

(a) Speed Deviation, $\omega_{21}$

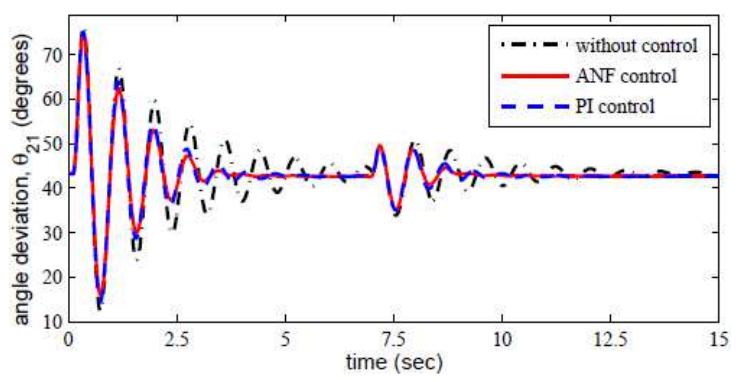

(b) Angle Deviation, $\theta_{21}$

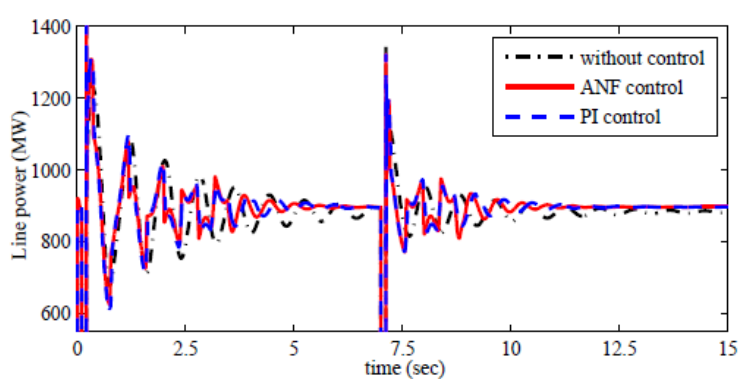

(c) Line Power Injected

Figure9. Scenario 2 Simulation Results

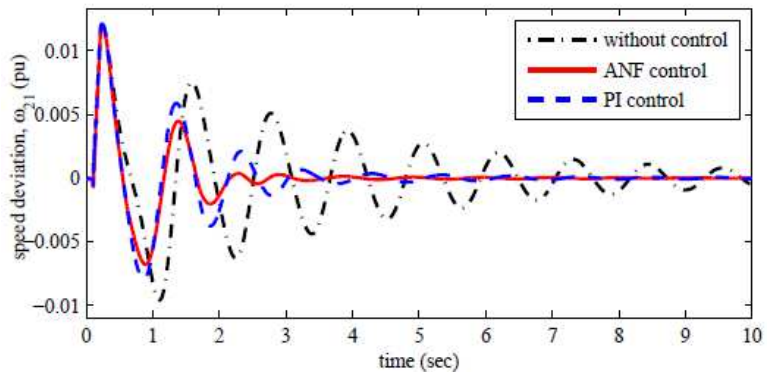

(a) Speed Deviation, $\omega_{21}$

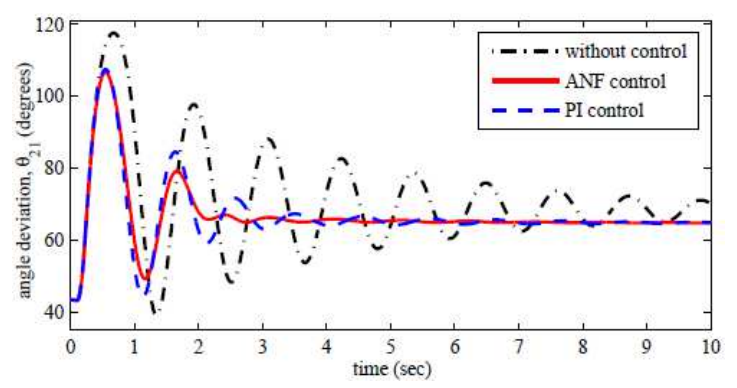

(b) Angle Deviation, $\theta_{21}$ 


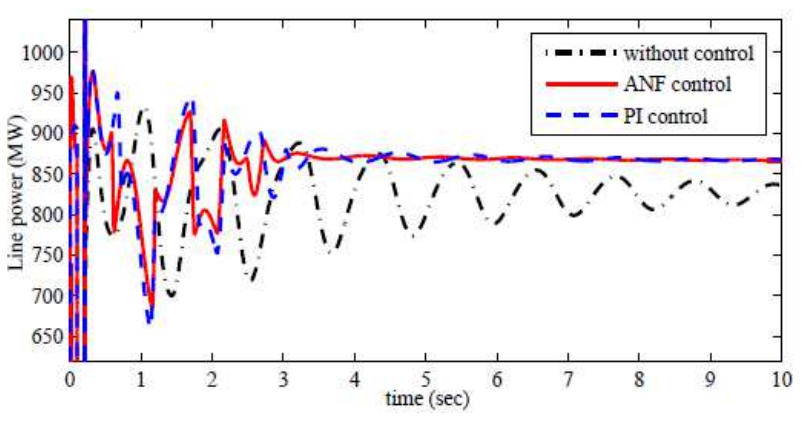

(c) Line Power Injected

Figure10. Scenario 3 Simulation Results

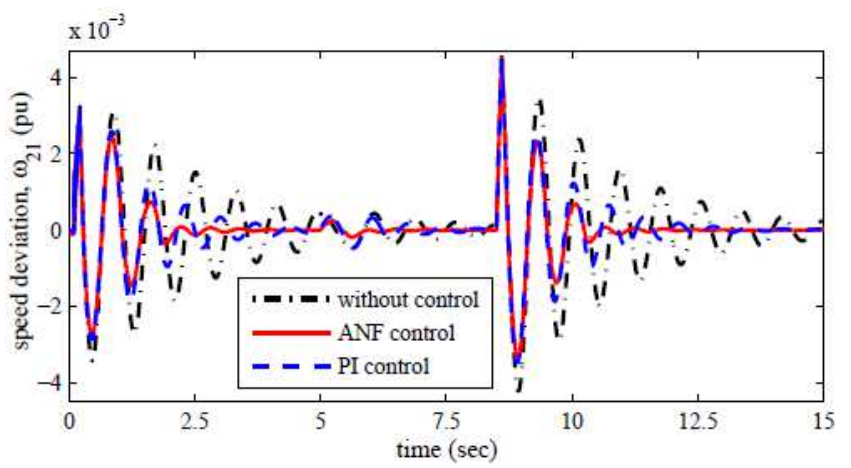

(a) Speed Deviation, $\omega_{21}$

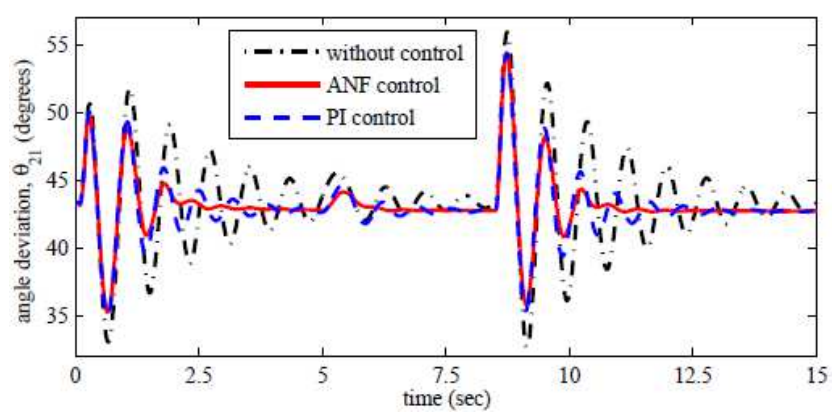

(b) Angle Deviation, $\theta_{21}$

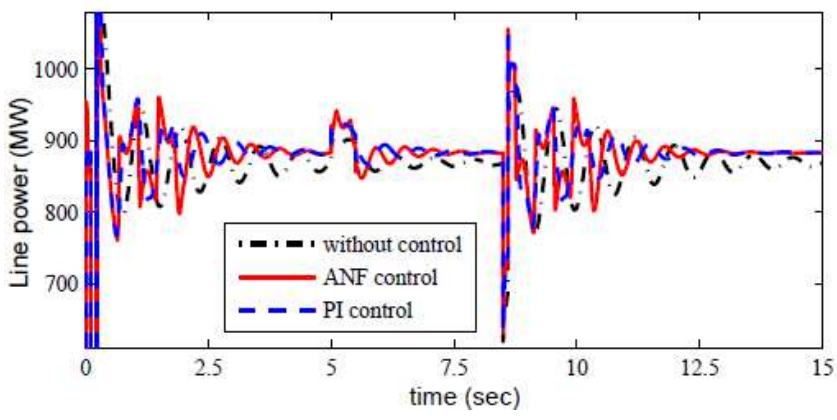

(c) Line Power Injected

Figure11. Scenario 4 Simulation Results

\subsection{Scenario 4}

To further examine the validation and robustness of proposed control strategy, series of faults are applied on the multi-machine test system. It comprises of self clearing, three-phase fault applied on line $\mathrm{L}_{3}$ at $\mathrm{t}=0.1 \mathrm{sec}$ for $115 \mathrm{sec}$ in addition with temporarily load outage (Load 1$)$ for $0.5 \mathrm{sec}$ and line outage $\left(\mathrm{L}_{2}\right)$ for $0.1 \mathrm{sec}$ at $\mathrm{t}=5 \mathrm{sec}$ and $\mathrm{t}=8.5 \mathrm{sec}$, respectively. Fig. 11 shows the superior ability of SVC-ANF control to effectively damp the system oscillations and restores the power system to its original steady-state. The settling time for speed deviation of SVC-ANF acquire a performance improvement of $21 \%$ and $26 \%$ as compared to SVC-PI and without control respectively, Fig. 11(a). It is clear from Fig. 11(b) that the rotor angle stabilizes itself to the original steady state when it undergoes various faults. The line power injected is shown in Fig. 11(c). It is revealed from above discussions, the proposed ANF strategy gives the freedom to tune the whole system and the control force can be initiated locally or globally depending on the system state. The major advantage of the ANF is that, it provides more design flexibility and increases the range of achievable objectives.

In order to optimize the performance of proposed ANF control, the integral of absolute error performance indices based on the (40) is calculated over the time interval, $0 \leq t \leq T_{s}$. The simulation time ' $T$ ' ' is appropriately selected to maximum span the transient response of the system [33].

$$
P_{A E}=\int_{0}^{T_{s}} t^{r} \sum_{2}^{n}|\Lambda| d t
$$

Where, $n$ shows the number of machines in the power system followed by $r=\{0,1\}$ for IAE and ITAE, respectively. Here, IAE examine the system performance in transient region while ITAE in steady-state. Smaller the value of $P_{A E}$, better is the system response in particular region.

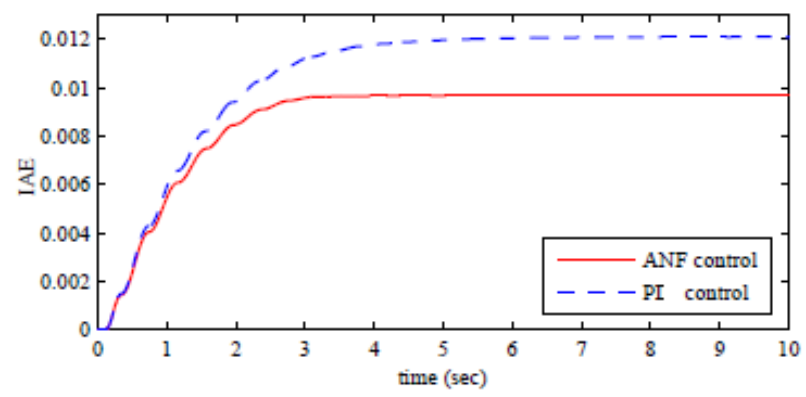

(a) IAE

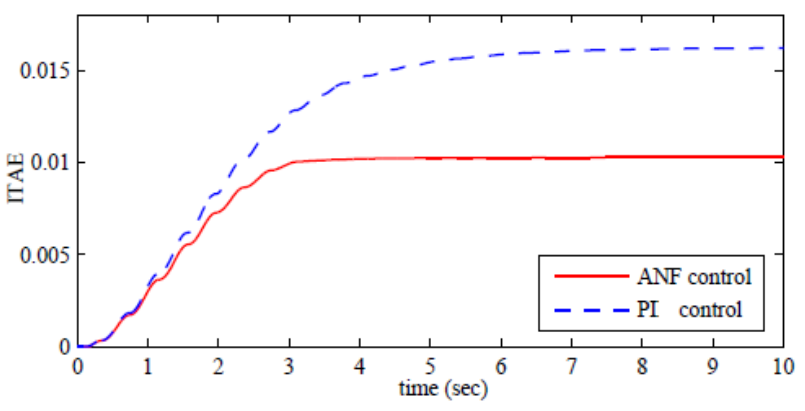

(b) ITAE

Figure12. Scenario 1 Performance Indices 


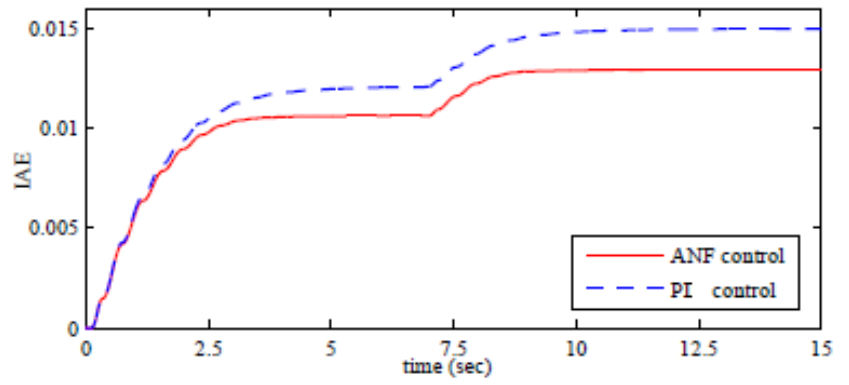

(a) IAE

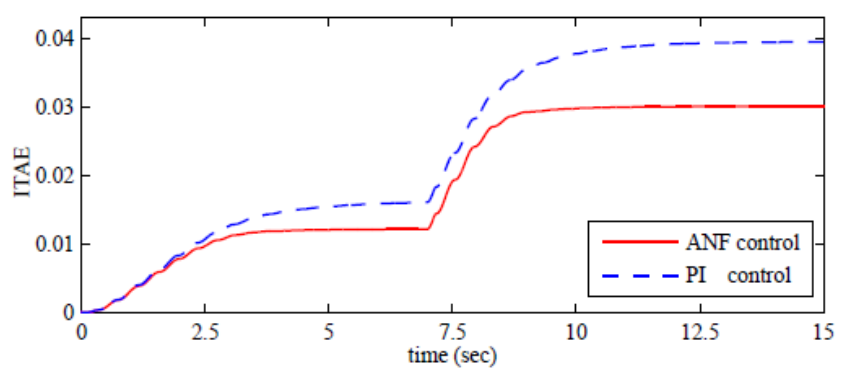

(b) ITAE

Figure13. Scenario 2 Performance Indices

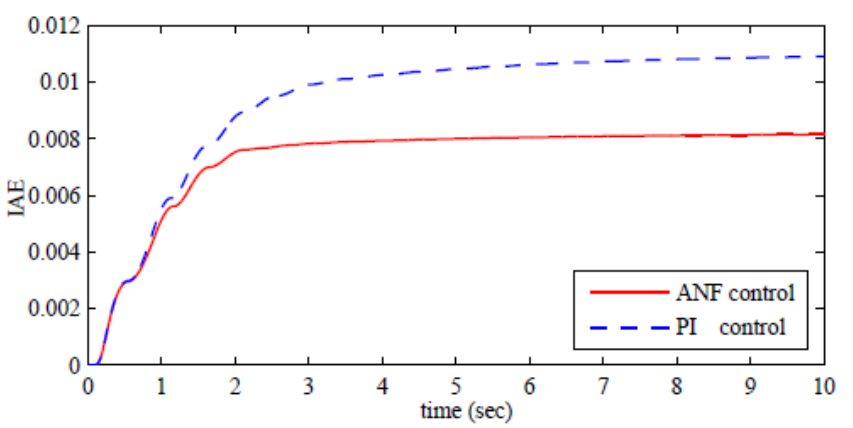

(a) IAE

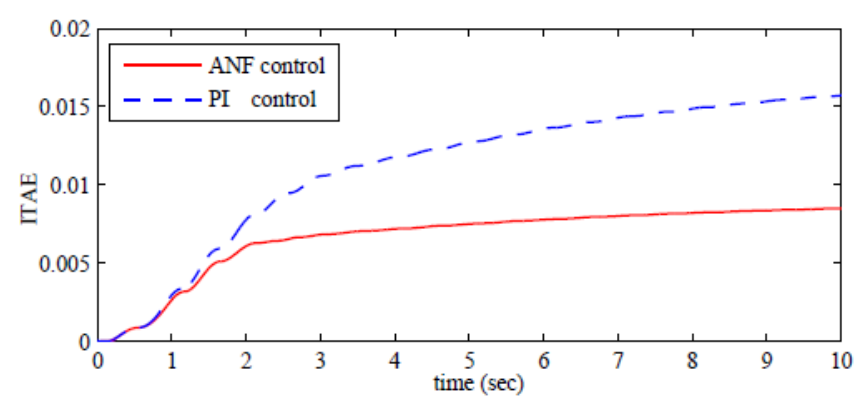

(b) ITAE

Figure14. Scenario 3 Performance Indices

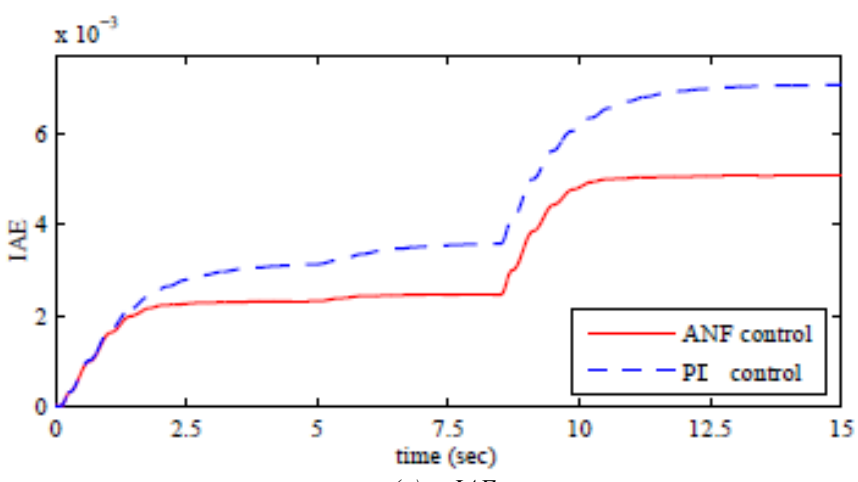

(a) IAE

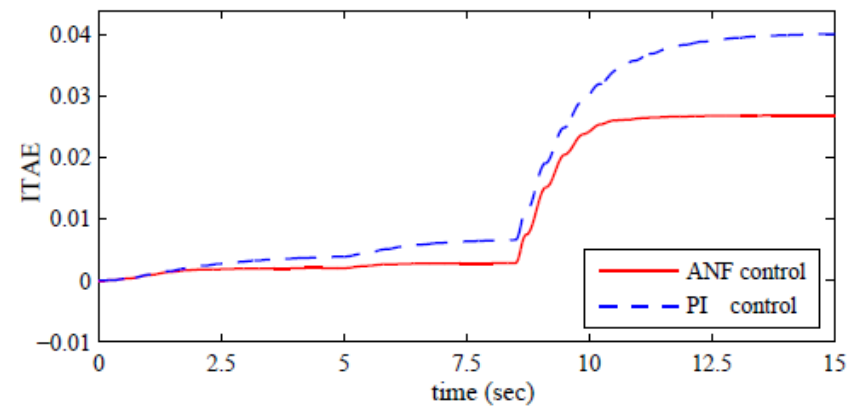

(b) ITAE

Figure15. Scenario 4 Performance Indices

Fig. 12 to Fig. 15 shows the improved behavior of ANF during both transient and steady-state regions. These results indicate that in case of ANF control, the value of ITAE is almost constant in steady-state resulting in zero steady-state error. Likewise, the results of IAE show the minimization of the overshoot during ANF control. Table 1 shows the performance indices comparative analysis for proposed ANF control with SVC-PI control. In scenario 1 and 2 the value of IAE for ANF control is reduced very much as compared to SVC-PI, showing much better performance in transient region.

Table1. Performance Index

\begin{tabular}{lllll}
\hline & $\begin{array}{l}\text { IAE } \\
\text { ANF }\end{array}$ & PI & ANF & PI \\
\hline Scenario 1 & 0.009667 & 0.01209 & 0.01027 & 0.01621 \\
Scenario 2 & 0.01292 & 0.01497 & 0.03005 & 0.0395 \\
Scenario 3 & 0.008147 & 0.0109 & 0.008486 & 0.01571 \\
Scenario 4 & 0.005062 & 0.007065 & 0.02686 & 0.04013 \\
\hline
\end{tabular}

\section{Conclusion}

In this paper ANF control has been successfully implemented as an SVC external control. The parameters of the proposed control are adjusted based on GD learning algorithm. To exhibit the capabilities of SVC external control, a multi-machine power system installed with SVC under various disturbances has been considered as a test system in MATLAB. To show the effectiveness of ANF 
control, simulation results are compared with PI control and without control. Results reveal that the designed SVC auxiliary control has a great potential to assure the robust performance in damping power system oscillations. It is also observed that the proposed strategies increase the robustness, efficiency, convergence and efficiency of the system. Also, the productivity and computational ability of the proposed ANF technique is better than the PI technique.

\section{References}

[1] M. Nikzad, S.S.S. Farahani and M.G. Naraghi, "Studying the performance of Static Var Compensator tuned based on simulated annealing in a multi-machine power system," American J. Scientific Res., vol. 23: pp. 73 - 82, 2011.

[2] P. Kundur, Power system stability and control, 2nd ed., USA: Mc Graw-Hill, 1993.

[3] P. M. Anderson and A.A. Fouad, Power System Control and Stability, 2nd ed., USA: Wiley- IEEE Press, 1997.

[4] X. Lei, E.N. Lerch and D. Povh, "Optimization and coordination of damping controls for improving system dynamic performance," IEEE Trans. Power Syst., vol. 16, $473-480,2001$

[5] E. V. Larsen and D. A. Swann, "Applying power system stabilizers, P-III, practical considerations," IEEE Trans. Power App. Syst., 1981, vol. 100, pp. 3034 - 3046, 1981

[6] J. G. Douglas and G. T. Heydt, "Power Flow Control and Power Flow Studies for Systems with FACTS Devices," IEEE Trans. Power Syst., vol. 13, 60 - 65, 1998.

[7] R. Majumder, B. C. Pal, C. Dufour, and P. Korba, "Design and real time implementation of robust FACTS controller for damping inter area oscillation," IEEE Trans. Power Syst., vol. 21, pp. $809-816,2006$.

[8] P. Rao, M. L. Crow and Z. Yang, "STATCOM control for power system voltage control applications," IEEE Trans. Power Delivery, vol. 15, pp. 1311 - 1317, 2000.

[9] H. Rahman, F. Rahman and H. Rashid, "Stability improvement of power system by using SVC with PID controller,” Int. J. Emerging Tech. Adv. Eng., vol. 2, 2012.

[10] L. Wang, "Comparative study of power system stabilizers, Static VAR Compensators and rectifier current regulators for damping of power system generator oscillations," IEEE Trans. Power Syst., vol. 8, pp. 613 - 619, 1993.

[11] S. C. Kapoor, "Dynamic stability of static compensator synchronous generator combination," IEEE Trans. Power App. Syst., vol. PAS-100, pp. $1694-1702,1981$.

[12] E. Z. Zhou, "Application of Static VAR compensators to increase power system damping," IEEE Trans. Power Syst., vol. 8, pp. $655-661,1993$.

[13] B. Pal and B. Chaudhuri, Robust control in power systems, New York, USA: Springer, 2005.

[14] Y. Chang and X. Zhen, "A novel SVC supplementary controller based on wide area signals," Electr. Power Syst. Res., vol. 77, pp. 1569 - 1574, 2007.
[15] P. K. Dash, S. Mishra, G. Panda, "Damping multimodal power system oscillations using a hybrid fuzzy controller for series connected FACTS devices," IEEE Trans. Power Syst., vol. 15, pp. $1360-1366,2000$.

[16] M. Kamari, et al. "Computational intelligence approach for SVC-PID controller in angle stability improvement," IEEE Conf. Power Eng. Opt., 2012.

[17] H. Rahman, R. Islam Sheikh and H. O. Rashid, "Stability Improvement of Power System by Using PI \& PD Controller," Comp. Tech. App. vol. 4, 111-118, 2013.

[18] N. Karpagam and D. Devaraj, "Fuzzy logic control of Static Var Compensator for Power System Damping," Int. Journal of Elect. Power and Energy Syst, vol. 2, no.2, pp. 105-111, 2009.

[19] M. P. Young, S. C. Myeon and Y. L. Kwang, "A neural network-based power system stabilizer using power flow characteristics," IEEE Trans. Energy Conv., vol. 11, no. 2, pp.435-441, 1996.

[20] J. Lu, M. H. Nehrir, D.A. Pierre,” A fuzzy logic based adaptive damping controller for Static VAR Compensator," Electr. Power Syst. Res., vol. 68, pp. 113 - 118, 2004.

[21] Y. Y. Hsu and L. H. Jeng, "Damping of subsynchronous oscillations using adaptive controllers tuned by artificial neural networks," IEE P. - Gener. Transm. D., vol. 142, no. 4, pp. $415-422,1995$.

[22] N. A. Arzeha, M. W. Mustafa, R. M Idris, "Fuzzy-based Static VAR Compensator controller for damping power system disturbances," IEEE Int. Conf. Power Eng. Opt., Malaysia, pp. 538 - 542, 2012.

[23] B. Changaroon, S. C. Srivastava, D. Thukaram, and S. Chirarattananon, "Neural network based power system damping controller for SVC," IEE P. - Gener. Transm. D., vol. 142, no. 4, pp. 370-376, 1999.

[24] T. Hiyama, W. Hubbi, and T. H. Ortmeyer, "Fuzzy logic control scheme with variable gain for static VAr compensator to enhance power system stability," IEEE Trans. Power Syst., vol. 14, pp. 186-191, February 1999.

[25] T. Hiyama, M. Mishiro, and H. Kihara, "Fuzzy logic switching of thyristor controlled braking resistor considering coodination with SVC," IEEE Trans. Power Delivery, vol. 10, pp. 2020-2026, October 1990.

[26] D. Z. Fang, Y. Xiaodong, T. S. Chung and K. P. Wong, "Adaptive Fuzzy-Logic SVC Damping Controller Using Strategy of Oscillation Energy Descent," IEEE Trans. Power Syst., vol. 19, no. 3, pp. 1414-1421, 2004.

[27] S. M. Hosseini, J. Olamaee and H. Samadzadeh, "Power Oscillations Damping by Static Var Compensator Using an Adaptive Neuro-Fuzzy Controller," 7th Int. Conf. Elect. Electron. Eng., vol. I-80, 2011.

[28] Li Wang, Dinh-Nhon Truong. Stability Enhancement of a Power System with a PMSG-based and a DFIG-based Offshore Wind Farms Using a SVC with an Adaptive-Network-based Fuzzy Inference System. IEEE Trans. Ind. Electron., vol. 60, no. 7, pp. 2799 - 2807, 2013.

[29] N. G. Hingorani, L. Gyugyi, Understanding FACTS: concepts and technology of flexible AC transmission systems, New York: IEEE Press, 2000. 
[30] H. F. Wang, "Phillips-Heffron model of power systems installed with STATCOM and applications," IEE P. - Gener. Transm. D., vol. 146 no. 5, pp. 521- 527, 1999.

[31] K. Ahad and M. V. Sohrforouzani. "Power system damping using fuzzy controlled facts devices," Int. J. Elect. Power, vol. 28 , no. 5, 349-357, 2006.
[32] Y. Xiao-dong, et al. “An adaptive SVC fuzzy controller for damping tie-link low frequency oscillation," Proc. of the Csee, vol. 1, 0-12, 2003.

[33] M. S. Tavazoie, "Notes on integral performance indices in fractional-order control systems," J. of Process Contr., vol. 20, no. 3, 285-291, 2010. 